Este libro forma parte del acervo de la Biblioteca Jurídica Virtual del Instituto de Investigaciones Jurídicas de la UNAM

\title{
NIÑAS, NIÑOS Y ADOLESCENTES EN PANDEMIA
}

\author{
Amaranta MANRIQUE DE LARA \\ Nashieli RAMÍREZ HERNÁNDEZ
}

\begin{abstract}
SUMARIO: I. Introducción. II. El impacto de COVID-19 en niñas, niños y adolescentes. III. La salud más allá del coronavirus. IV. Niñas, niños y adolescentes como protagonistas del distanciamiento. V. Efectos psicosociales del encierro. VI. Conclusiones: la urgencia de lo inmediato.
\end{abstract}

\section{INTRODUCGIÓN}

Desde el inicio de la pandemia global de COVID-19, las niñas, niños y adolescentes ${ }^{1}$ han esquivado los peores efectos de salud que provoca la infección por SARS-GoV-2. La proporción de casos es menor y, cuando existe enfermedad, ésta es considerablemente menos severa. ${ }^{2}$ Las infancias y adolescencias no son entonces la cara de esta crisis sanitaria y, tal vez por lo mismo, se han dejado de lado. Sin lugar a duda, el coronavirus llegó a exacerbar las desigualdades y arrojar luz sobre las fallas estructurales que apenas sostienen la organización social actual a nivel global. En este sentido, también se ha puesto en evidencia la prevalencia de una visión adultocéntrica en nuestras sociedades, donde las niñas, niños y adolescentes son vistos como un valor futuro y sus voces ignoradas en el presente.

Aunque no sea directamente provocado por el virus, lo cierto es que las infancias y adolescencias están en riesgo de convertirse en una de las

1 De acuerdo con la Ley General de los Derechos de Niñas, Niños y Adolescentes, son niñas y niños los menores de doce años, y adolescentes las personas de entre doce años cumplidos y menos de dieciocho años de edad (artículo 5o.). Consultar en: http://wrwre.dof.gob.mx/ nota_detalle.php? codigo $=5374143$ Efecha $=04 / 12 / 2014$.

2 Ludvigsson, Jonas F., "Systematic review of COVID-19 in children shows milder cases and a better prognosis than adults", Acta Paediatrica, Noruega, vol. 109, núm. 9, 2020, pp. 1088-1095, DOI:10.1111/apa.15270. 
Este libro forma parte del acervo de la Biblioteca Jurídica Virtual del Instituto de Investigaciones Jurídicas de la UNAM www.juridicas.unam.mx

mayores víctimas de esta crisis, ya que las respuestas de los gobiernos y las medidas de mitigación implementadas afectan de manera profunda - y en algunos casos, quizás permanente - la vida de estos individuos que conforman el 30.2\% de la población mundial. ${ }^{3}$ Los impactos indirectos de la pandemia tienen ya efectos observables que impiden el pleno ejercicio de los derechos de las infancias y adolescencias, incluidos el derecho a la salud, a la educación, a la integridad personal, a la identidad, a una vida libre de violencia, al esparcimiento, entre otros. Además, aunque todas las niñas, niños y adolescentes se ven afectados, un enfoque interseccional reconoce que existe un impacto diferenciado dependiendo del contexto. Variables como la edad, género, discapacidad, situación socioeconómica o la acumulación de más de una de estas capas de vulnerabilidad ${ }^{4}$ resulta en afectaciones en mayor o menor medida. De esta manera, son las infancias y adolescencias que de por sí son vulneradas, marginadas y violentadas quienes cargarán el mayor peso de los efectos negativos de esta pandemia.

La continua implementación de medidas de mitigación, así como la crisis económica que se viene gestando y que alterará la vida de miles de familias, vuelven urgente el reconocimiento y visibilización de las realidades de niñas, niños y adolescentes. Así como se ha incitado a las naciones a velar por el respeto de los derechos humanos incluso frente a medidas excepcionales a nombre del bien común, los esfuerzos por controlar la crisis sanitaria también deben considerar el efecto diferenciado sobre este grupo etario como un grupo de atención prioritaria, guardando el interés superior de niñas, niños y adolescentes como consideración primordial.

En este escrito, nos proponemos iniciar una reflexión sobre las infancias y adolescencias durante la pandemia de COVID-19, así como los efectos a corto plazo que ésta está teniendo en sus vidas. ${ }^{5}$ En un inicio hablaremos sobre el derecho a la salud de niñas, niños y adolescentes durante la

3 Organización de las Naciones Unidas, Departamento de Asuntos Económicos y Sociales, División de Población, Perspectivas de la Población Mundial 2019, 2019, datos personalizados obtenidos de la página web, https://population.un.org/wpp/DataQuery/.

4 Luna, Florencia, "Vulnerabilidad: la metáfora de las capas", Furisprudencia Argentina, vol. 4, núm. 2, 2008, pp. 60-67.

5 El análisis que se presenta a continuación tiene como fuente fundamental el informe nacional de la consulta \#InfanciasEncerradas, realizada por la Comisión de Derechos Humanos de la Ciudad de México. Más de 40 mil niñas, niños y adolescentes de las 32 entidades federativas expresaron sus vivencias durante el confinamiento, siendo la consulta en sí misma un ejemplo de satisfacción del derecho a la participación de las infancias y adolescencias. Dicho informe puede consultarse en: https://cdhcm.org.mx/wp-content/uploads/2020/07/Infancias_encerradas_Nacional.pdf. 
Este libro forma parte del acervo de la Biblioteca Jurídica Virtual del Instituto de Investigaciones Jurídicas de la UNAM

pandemia, en cuanto a la infección por SARS-GoV-2 y más allá de ésta. Posteriormente, nuestro enfoque será sobre cómo el cierre de escuelas - la medida que instrumentaliza el distanciamiento en este grupo etario - tiene un impacto global que incluye efectos sobre el aprendizaje, el bienestar psicosocial, y la salud mental.

\section{EL IMPACTO DE GOVID-19 EN NIÑAS, NIÑOS Y ADOLESCENTES}

Como ya mencionamos, la infección por coronavirus no representa riesgos considerables para niñas, niños y adolescentes, comparado con la severidad que puede llegar a tener la enfermedad en adultos. Sin embargo, esto no significa que no sean susceptibles a SARS-CoV-2, o que su experiencia con la enfermedad sea menos importante. La subestimación de la relevancia de este virus en este grupo etario ha llevado a un rezago de información específica - clínica, epidemiológica y científica - a nivel mundial. En efecto, son pocos los países que están proporcionando información desagregada por edad, por lo menos en América Latina. ${ }^{6} \mathrm{Al}$ mismo tiempo, la falta de información retroalimenta la percepción de irrelevancia.

México es uno de los países que sí reporta datos desagregados de COVID-19, de los cuales se puede obtener información preocupante. Por ejemplo, a pesar de las medidas de confinamiento y el cierre de escuelas en específico, los casos confirmados de niñas, niños y adolescentes ha ido incrementando constante y considerablemente. El número de casos se ha casi duplicado en un mes, entre el 6 de julio ${ }^{7}$ y el 6 de agosto, ${ }^{8}$ llegando a 11,828 casos. Es importante destacar que este comportamiento es más claro en adolescentes, cuya tasa de incidencia ha aumentado $17.16 \%$ desde el 12 de abril, mientras que en el grupo de primera infancia creció $10.56 \%$ en el

6 Convergencia para la Acción, "Situación general del COVID-19 en países del proyecto «Convergencia para la Acción»", Chile, 28 de julio de 2020, disponible en: http:// convergenciaparalaaccion.org/wp-content/uploads/2020/07/LAT-28-de-julio-2020.pdf.

7 Sistema Nacional de Protección de Niñas, Niños y Adolescentes, "COVID-19 Niñas, Niños y Adolescentes", México, 7 de julio de 2020, disponible en: https://onedrive.live.com/?au thkey $=\% 21 \mathrm{AMfif0} \% 5 \mathrm{FcOQyoRps}$ Ecid $=34 \mathrm{FA} 594 \mathrm{CF} 22743 \mathrm{CDE} i \mathrm{~d}=34 \mathrm{FA} 594 \mathrm{CF} 22743 \mathrm{CD} \% 212$ 1812 EparId $=34 F A 594 C F 22743 C D \% 2121331 \Xi_{0}=$ OneUp.

8 Sistema Nacional de Protección de Niñas, Niños y Adolescentes, "COVID-19 Niñas, Niños y Adolescentes", México, 7 de agosto de 2020, disponible en: https://onedrive.live. com/?authkey $=\% 21$ AMfifo_cOQyoRps\&cid $=34 F A 594 C F 22743$ CDEid $=34 F A 594 C F 22743 C D \%$ 2122297 \&parId=34FA594CF22743CD $\% 2121331$ \&o=OneUp. 
Este libro forma parte del acervo de la Biblioteca Jurídica Virtual del Instituto de Investigaciones Jurídicas de la UNAM

mismo periodo. ${ }^{9}$ De manera similar, ha habido 187 defunciones de menores de edad desde el 13 de abril que se registró la primera al 6 de agosto $;^{10}$ éstas aumentaron 1.72 veces desde el 6 de julio cuando habían $109 .{ }^{11}$ De estas defunciones registradas por COVID-19, el 48\% fueron mujeres y preocupa que más del $26 \%$ fueran niñas y niños en primera infancia. ${ }^{12}$ También cabe señalar que de las defunciones registradas, cuatro tenían adscripción indígena, equivalente al $2.13 \% .^{13}$

Aunque sí hay información específica, considerando que aquí la aplicación de pruebas de COVID-19 está ligada a valoraciones clínicas, ${ }^{14} \mathrm{y}$ este grupo etario generalmente presenta casos leves o asintomáticos, es probable que la subestimación de casos en niñas, niños y adolescentes sea mayor que en otros grupos. Esta falta de pruebas en infancias y adolescencias es consistente en otros países, ${ }^{15}$ y limita el análisis epidemiológico en este grupo etario a nivel global. De igual manera, hay poca información sobre la definición de casos y criterios clínicos, que podrían ser distintos a los de la enfermedad en adultos, ${ }^{16}$ así como falta de estrategias de manejo clínico y tratamiento específicas para pacientes pediátricos. ${ }^{17}$ Por ejemplo, un porcentaje pequeño de pacientes pediátricos positivos a COVID-19 de-

\section{Idem. \\ 10 Idem.}

11 Sistema Nacional de Protección de Niñas, Niños y Adolescentes, "COVID-19 Niñas, Niños y Adolescentes", México, 7 de julio de 2020, disponible en: https://onedrive.live.com/?au thkey $=\% 21 \mathrm{AMffo} \% 5 \mathrm{Fc} O$ QyoRps Ecid=34FA594CF22743CDEid=34FA594CF22743CD $\% 212$ 1812 EparId=34FA594CF22743CD\%2121331 E̊o = OneUp.

12 Sistema Nacional de Protección de Niñas, Niños y Adolescentes, "COVID-19 Niñas, Niños y Adolescentes", México, 7 de agosto de 2020, disponible en: https://onedrive.live. com/?authkey=\%21AMffo_cOQyoRpsÉcid=34FA594CF22743CDEid =34FA594CF22743CD\% 2122297 EparId=34FA594CF22743CD\%2121331 E०o=OneUp.

13 Idem.

14 Secretaría de Salud, "Lineamientos para la prevención, detección y atención de covid-19 en niñas, niños y adolescentes (NNA)", México, 21 de mayo de 2020, disponible en: https://coronavirus.gob.mx/wp-content/uploads/2020/05/Lineamientos_prevencion_deteccion_atencion_COVID_NNA-1.pdf.

15 Curley, Christopher, "What a Lack of COVID-19 Testing Means for Children and Reopening Schools", Healthline, 10 de julio de 2020, disponible en: https://wrwe.healthline.com/ health-news/lack-of-covid-19-testing-children-returning-to-school.

16 Xia, Wei et al., "Clinical and CT features in pediatric patients with COVID-19 infection: Different points from adults", Pediatric pulmonology, vol. 55, núm. 5, 2020, pp. 1169-1174, DOI:10.1002/ppul.24718.

17 Kelvin, Alyson A. y Halperin, Scott, "COVID-19 in children: the link in the transmission chain", The Lancet. Infectious diseases, vol. 20, núm. 6, 2020, pp. 633 y 634, DOI:10.1016/ S1473-3099(20)30236-X. 
Este libro forma parte del acervo de la Biblioteca Jurídica Virtual del Instituto de Investigaciones Jurídicas de la UNAM

sarrolla un síndrome inflamatorio multisistémico que puede llegar a ser fatal y que de momento se desconocen los posibles efectos a largo plazo por el daño al tejido pulmonar. ${ }^{18}$

No recolectar datos específicos sobre las infancias y adolescencias significa que se invisibilizan sus situaciones y que haya discriminación, ya que no se cuentan con los elementos suficientes para protegerles y garantizar su derecho a la salud. Además, tener información concreta sobre el papel de niñas, niños y adolescentes en la cadena de transmisión del virus es indispensable para implementar estrategias basadas en evidencia y proporcionales que protejan la salud comunitaria. En este sentido, sería recomendable desarrollar proyectos de investigación enfocados en este grupo etario. Por ejemplo, el proyecto HEROS ${ }^{19}$ del Instituto Nacional de Alergias y Enfermedades Infecciosas en Estados Unidos dará seguimiento a menores de edad y sus familias por un periodo inicial de seis meses para determinar la prevalencia del estatus de portador y el desarrollo de anticuerpos, con el propósito de informar mejores políticas de salud pública que involucren a estos grupos, como el cierre de escuelas o servicios de cuidado infantil.

\section{LA SALUD MÁS ALLÁ DEL CORONAVIRUS}

A pesar de la falta de datos específicos, de todos modos podemos observar que la infección por coronavirus no tiene un impacto directo tan grave sobre la salud de este grupo de edad en comparación con otros. Sin embargo, eso no significa que la pandemia no implique un impacto indirecto considerable sobre el ejercicio del derecho a la salud de infancias y adolescencias. De manera amplia, las vidas de niñas, niños y adolescentes se ven afectadas tanto por la presión sobre sistemas de salud de por sí frágiles, como por los efectos de la crisis económica sobre los ingresos familiares.

Desde la primera infancia, se espera ver un incremento a la tasa de mortalidad de menores de cinco años en países de ingresos medio y bajo, por una reducción de la cobertura de salud de rutina. ${ }^{20}$ Del escenario más opti-

18 Riphagen, Shelley et al., "Hyperinflammatory shock in children during COVID-19 pandemic", Lancet, vol. 395, núm. 10237, 2020, pp. 1607 y 1608, DOI:10.1016/S01406736(20)31094-1.

19 National Institute of Allergy and Infectious Diseases, "COVID-19: Human Epidemiology and Response to SARS-CoV-2 (HEROS)", Estados Unidos, 5 de mayo de 2020, disponible en: https://wrerc.clinicaltrials.gov/ct2/show/study/NCT04375761.

20 Roberton, Timothy et al., "Early estimates of the indirect effects of the COVID-19 pandemic on maternal and child mortality in low-income and middle-income coun- 
Este libro forma parte del acervo de la Biblioteca Jurídica Virtual del Instituto de Investigaciones Jurídicas de la UNAM

mista al más catastrófico, un estudio calcula un incremento de entre el 9.8 y el $44.7 \%$ de muertes infantiles mensuales. ${ }^{21}$ La importancia de la atención a la salud y el seguimiento del desarrollo durante los primeros meses a años de vida deben tenerse presentes durante la respuesta a la crisis sanitaria, asegurando siempre el acceso a estos servicios para niñas y niños. En este sentido, también es importante asegurar el acceso a una alimentación sana y a servicios como agua para infancias y adolescencias, independientemente de la situación económica de sus familias y cómo se vea ésta afectada por la pandemia.

De manera similar, se está viendo un efecto de la pandemia sobre la medicina preventiva, incluyendo los esquemas de vacunación. La inmunización contra enfermedades como el sarampión y la poliomielitis ha disminuido a nivel global en estos meses. Esto se debe no sólo a la interrupción de la movilidad individual por las políticas de confinamiento, sino también a la reducción de la movilidad internacional comercial. El Fondo de las Naciones Unidas para la Infancia (UNICEF) ha documentado una disminución de entre 70 y $80 \%$ de los envíos de vacunas, lo que ya afecta al menos a 26 países y pone en riesgo a muchos otros cuyas reservas podrían terminarse en el mediano plazo. ${ }^{22}$ En efecto, en México se ha detectado un rezago de aproximadamente el 20\%; es decir, 700000 niñas y niños afectados por un retraso en alguna dosis de su esquema de vacunación. ${ }^{23}$ El Instituto Mexicano del Seguro Social espera poder regularizar este servicio antes de que termine el año; sin embargo, esto seguiría dejando desprotegidos a niñas y niños que requieran inmunización contra enfermedades como el rotavirus cuyas dosis deben administrarse a más tardar a los ocho meses de edad. ${ }^{24}$ En cuanto a la vacuna contra la influenza estacional, se ha dicho que por lo menos en la Ciudad de México estarán todas las dosis aseguradas. ${ }^{25}$ Habrá

tries: a modelling study", The Lancet. Global Health, vol. 8, núm. 7, 2020, pp. E901-E908, DOI:10.1016/S2214-109X(20)30229-1.

21 Idem.

22 Fondo de las Naciones Unidas para la Infancia, "Geneva Palais briefing note on the impact of COVID-19 mitigation measures on vaccine supply and logistics", Ginebra, Suiza, 1 de mayo de 2020, disponible en: https://wrerw.uniceforg/press-releases/geneva-palais-briefingnote-impact-covid-19-mitigation-measures-vaccine-supply-and.

23 Valadez, Blanca, "Covid-19 retrasa vacunación de 700 mil niños", Milenio, Ciudad de México, México, 27 de julio de 2020, disponible en: https://wrere.milenio.com/ciencia-y-salud/ coronavirus-imss-detecta-retraso-vacunacion-700-mil-ninos.

24 Idem.

25 Wong, Alma Paola, "CdMx adelantará periodo de vacunación de influenza por coronavirus", Milenio, Ciudad de México, México, 22 de julio de 2020, disponible en: https:// wrwemilenio.com/politica/comunidad/influenza-cdmx-adelantara-etapa-vacunacion-coronavirus. 
Este libro forma parte del acervo de la Biblioteca Jurídica Virtual del Instituto de Investigaciones Jurídicas de la UNAM

que dar seguimiento a que se cumpla el acceso a niños y niñas en primera infancia, ya que sí son un grupo en riesgo de sufrir complicaciones por esta infección respiratoria.

En general, el confinamiento de niñas, niños y adolescentes, y/o la negación a asistir a centros de salud por miedo al contagio deja desprotegidas a las y los pacientes pediátricos. Por ejemplo, la falta de movilidad afecta a infancias y adolescencias con condiciones preexistentes que necesitan monitoreo o rehabilitación que no puede ofrecerse a distancia. Aunque la pandemia ha vuelto urgente la discusión sobre la provisión de servicios de salud por telemedicina, la atención pediátrica a distancia necesitaría tomar en cuenta consideraciones específicas. ${ }^{26}$, Por ejemplo, el monitoreo del correcto desarrollo necesita seguimiento directo, además de que limitar el nivel de interacción verbal y no verbal complicaría la buena atención a pacientes pediátricos que recién aprenden a comunicarse.

Otro efecto indirecto de la pandemia se ve en la salud sexual y reproductiva de adolescentes. Durante el tiempo de confinamiento se ha visto un aumento del 20\% en los embarazos de adolescentes en México, y se estima que para el próximo año habrá más de 145 mil embarazos de este tipo en este grupo etario. ${ }^{27}$ Esta situación puede atribuirse en parte a que las adolescentes no están pudiendo acudir a solicitar anticonceptivos por la disminución de la movilidad. Sobre este tema también debe mencionarse que de los embarazos en menores de 14 años, se calcula que por lo menos el $20 \%$ son producto de violaciones. ${ }^{28} \mathrm{El}$ incremento de la violencia durante el confinamiento se discute en otro capítulo de este libro, pero vale la pena hacer énfasis que las infancias y adolescencias también son víctimas de este fenómeno y que deben existir mecanismos para asegurar su protección y bienestar. Además, en el tema específico de los embarazos no deseados, es también un tema del ejercicio del derecho a la salud de niñas y adolescentes poder acceder a métodos de interrupción seguros y no revictimizantes.

La realidad es que esta pandemia va a afectar la salud y el bienestar de niñas, niños y adolescentes, independientemente de si se contagien de

26 Rosenthal, Marjorie S., "The New Language of Telehealth", The New York Times, Estados Unidos, 5 de mayo de 2020, disponible en: https://wrere.nytimes.com/2020/05/05/well/ live/doctors-patients-mother-baby-pediatrics-telemedicine-computers.html.

Hatcher, Cyndie, "As telemedicine takes hold, what are doctors like me missing?", The Boston Globe, Estados Unidos, 3 de junio de 2020, disponible en: https://wrere.bostonglobe. com/2020/06/03/magazine/telemedicine-takes-hold-what-are-doctors-like-me-missing/.

27 Rodríguez, Gabriela, "Alarmante aumento del embarazo en adolescentes en México", entrevista por Mónica Garza, ADN40, 7 de agosto de 2020.

28 Idem. 
Este libro forma parte del acervo de la Biblioteca Jurídica Virtual del Instituto de Investigaciones Jurídicas de la UNAM

COVID-19 o no. Como sucede con la población adulta, es inevitable hacer compromisos cuando el sistema de salud se ve abrumado en todos los niveles. Aunque se requieren sacrificios de todos los miembros de la sociedad para lograr mitigar los daños de este virus, a continuación discutiremos por qué consideramos que a este grupo etario se le han pedido más sacrificios que a otros.

\section{NIÑAS, NIÑOS Y ADOLESGENTES COMO PROTAGONISTAS DEL DISTANCIAMIENTO}

Durante la pandemia, el distanciamiento social ha sido la fórmula de mitigación más utilizada a nivel global. Si consideramos que la población infantil y adolescente comprende casi la tercera parte de la población mundial, es evidente que el éxito de esta medida descansa en este grupo etario, a quien se le ha obligado a salir de los espacios públicos. En particular, el cierre de espacios escolares - medida indispensable para el distanciamiento de niñas, niños y adolescentes - ha puesto en riesgo la garantía del derecho a la educación.

A nivel global, se ha optado por suspender la oferta educativa presencial. A principios de febrero, incluso antes de que la Organización Mundial de la Salud (OMS) declarara oficialmente la pandemia, el cierre de escuelas impactaba ya al $0.1 \%$ de la población estudiantil mundial, debido al cierre nacional en Mongolia y el cierre focalizado en China. ${ }^{29}$ Para finales de marzo, eran 179 los países con cierres a nivel nacional, incluyendo a México, y a mediados de abril 191 países reportaron la clausura total de la oferta educativa presencial. ${ }^{30}$ En el punto más alto de cierre de escuelas, se calcula que aproximadamente el 90\% de la población estudiantil global se vio afectada. ${ }^{31}$ Entre estos, seis de cada diez niñas y niños no han recibido educación de ningún tipo debido en parte a la brecha digital - falta de herramientas tecnológicas como computadoras, tabletas, teléfonos inteligentes, etcétera, así como falta de acceso a internet- . No es sorpresa que esta falta de escolarización se vea en mayor medida en países de desarrollo humano bajo. ${ }^{32}$

\footnotetext{
29 Organización de las Naciones Unidas para la Educación, la Ciencia y la Cultura, "Seguimiento mundial de los cierres de escuelas causados por el COVID-19", disponible en: https://es.unesco.org/covid19/educationresponse.

30 Idem.

31 Idem.

32 Programa de las Naciones Unidas para el Desarrollo, COVID-19 and Human Development: Assessing the Crisis, Envisioning the Recovery, Nueva York, 2020, disponible en: http://hdr. undp.org/sites/default/files/covid-19_and_human_development_0.pdf.
} 
Este libro forma parte del acervo de la Biblioteca Jurídica Virtual del Instituto de Investigaciones Jurídicas de la UNAM

Este fenómeno de desigualdad lo podemos observar de manera más local, donde la brecha educativa entre poblaciones urbanas y rurales también tenderá a acentuarse. Las propuestas de educación a distancia en México se dan en un contexto donde sólo cuatro de cada diez tienen acceso fluido a internet. ${ }^{33}$ Aun si la modalidad a distancia se plantea con la televisión ${ }^{34}$ nos estaremos enfrentando a problemas de desigualdad, porque existen zonas donde sólo se puede acceder incluso a los canales abiertos si se cuenta con televisión de paga porque la antena no es suficiente.

Parece que nos encontramos no sólo frente a una crisis sanitaria y económica, sino también frente a una emergencia educativa. Pero más allá de los desafíos tecnológicos que presenta la educación durante la pandemia - el avance hacia las nuevas tecnologías en lo educativo era un proceso que se tenía que ir dando de cualquier manera - tenemos que también atender las implicaciones de la falta de la escuela como el centro de la vida social de las niñas, niños y adolescentes; el espacio donde se construyen sus relaciones y vínculos fuera del ámbito familiar.

La generación de conocimiento es sólo uno de los propósitos de la escuela. En realidad, el tiempo efectivo de clases es mínimo en la educación presencial, ${ }^{35}$ aunque la carga de trabajo se ha incrementado drásticamente durante la transición a la educación a distancia. La función social de la escuela es la que tenemos que trabajar por fortalecer y preservar. Lo que hace la vida social durante la primera infancia es el entorno cercano, pero después las niñas y niños tienen que ir aprendiendo a vivir en sociedad. La adquisición de herramientas sociales, e incluso la formación inicial de ciudadanía, se da a partir de la relación con los otros. El centro de la vida de las niñas, niños y adolescentes a nivel de primaria, secundaria y media superior es la escuela. Las actividades escolares son una válvula también que resuelve cotidianidades. Es en este aspecto en el que la ausencia de la escuela está teniendo y va tener su mayor impacto, y hay que empezar a plantearnos maneras en las que podemos intensificar la vinculación de infancias y adolescencias con sus pares durante el confinamiento.

33 Instituto Federal de Telecomunicaciones, "Encuesta Nacional sobre Disponibilidad y Uso de Tecnologías de la Información en los Hogares, 2015 (Comunicado 27/2016)", 14 de marzo de 2016, disponible en: http://wrere.ift.org.mx/comunicacion-y-medios/comunicados-ift/es/ encuesta-nacional-sobre-disponibilidad-y-uso-de-tecnologias-de-la-informacion-en-los-hogares-2015.

34 Infobae, "Esteban Moctezuma explicó cómo será el regreso a clases para el Ciclo Escolar 2020-2021 de la SEP", México, 3 de agosto de 2020, disponible en: https://wrwreinfobae. com/america/mexico/2020/08/03/esteban-moctezuma-explico-como-sera-el-regreso-a-clases-para-elciclo-escolar-2020-2021-de-la-sep/.

35 Razo Pérez, Ana Elizabeth, "Tiempo de aprender: El aprovechamiento de los periodos en el aula", Revista Mexicana de Investigación Educativa, vol. 21, núm. 69, 2016, pp. 61 1-639. 
Este libro forma parte del acervo de la Biblioteca Jurídica Virtual del Instituto de Investigaciones Jurídicas de la UNAM

Un último aspecto relacionado con el cierre de escuelas es que impacta de forma directa en la posibilidad de incorporación de niñas y niños al trabajo infantil. Este factor contribuye también al crecimiento de las desigualdades de género en las expectativas del tipo de trabajo que las niñas pueden realizar, como trabajo de casa o en el campo. Finalmente, los trabajos de cuidado que recaen sobre niñas y niños les exponen a riesgos de contraer enfermedades, en especial cuando se encargan de familiares enfermos. ${ }^{36}$

\section{EFECTOS PSICOSOCIALES DEL ENCIERRO}

La vida cotidiana de millones de niñas, niños y adolescentes se transformó de manera radical, de un día a otro el espacio público despareció, y con él, el vínculo con sus pares y otros adultos que le son significativos. Sumando a lo anterior un contexto de incertidumbre y miedo, con limitadas respuestas de contención de parte de sus cuidadores. Las medidas de confinamiento han sido un caldo de cultivo para la generación de situaciones que ponen en riesgo su equilibrio psicosocial.

Durante el periodo de atención a las medidas de prevención derivadas de la pandemia por COVID-19, diversos organismos internacionales como $\mathrm{UNICEF}^{37}$ y la $\mathrm{OMS}^{38}$ han señalado la emergencia de problemas en el ámbito de la salud mental de niñas, niños y adolescentes. Estas preocupaciones surgen del entendimiento integral de la salud como "un estado de completo bienestar fisico, mental y social, y no solamente la ausencia de afecciones o enfermedades". ${ }^{39}$

De acuerdo con la OMS y en consonancia tanto con el propio concepto de salud integral que este organismo promueve, como con el contenido del derecho a la salud tal como ha sido interpretado por el Comité de Dere-

36 Alianza para la Protección de la Niñez y Adolescencia en la Acción Humanitaria, "Nota técnica: COVID-19 y trabajo infantil", 6 de mayo de 2020, disponible en: https://alliancecpha.

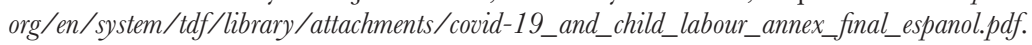

37 Fondo de las Naciones Unidas para la Infancia, "How to protect your family's mental health in the face of coronavirus disease (COVID-19)", 26 de marzo de 2020, disponible en: https://wrwe.uniceforg/coronavirus/how-protect-your-familys-mental-health-face-coronavirus-diseasecovid-19.

38 Agencia EFE, "La OMS advierte que la COVID-19 afectará a la salud mental futura", 14 de mayo de 2020, disponible en: https://wrere.efe.com/efe/espana/sociedad/la-oms-advierte-que-covid-19-afectara-a-salud-mental-futura/10004-4245936.

39 Organización Mundial de la Salud, "Constitución de la Organización Mundial de la Salud”, Documentos Básicos, 48a. ed., Italia, Organización Mundial de la Salud, 2014, pp. 1-21, disponible en: https://apps.who.int/gb/bd/PDF/bd48/basic-documents-48th-edition-sp.pdf. 
Este libro forma parte del acervo de la Biblioteca Jurídica Virtual del Instituto de Investigaciones Jurídicas de la UNAM

chos Económicos, Sociales y Culturales de la Organización de las Naciones Unidas,$^{40}$ la salud mental debe de ser considerada más allá de la ausencia de trastornos mentales para ser observada como una posibilidad de bienestar. No puede hablarse de un estado de salud general si la salud mental está comprometida. En este sentido, es indispensable analizar cómo es que la situación de crisis actual puede estar afectando la salud mental de niñas, niños y adolescentes como grupo de atención prioritaria.

Ejercicios como la consulta \#InfanciasEncerradas nos pueden dar esta clase de indicios. Ésta fue realizada por la Comisión de Derechos Humanos de la Ciudad de México para conocer las vivencias de infancias y adolescencias durante el confinamiento, obteniendo datos de las 32 entidades federativas para hacer un análisis a nivel nacional. Como mencionamos anteriormente, recolectar datos específicos sobre este grupo etario es indispensable para visibilizar sus situaciones particulares y poder diseñar políticas públicas mejor informadas.

Según los resultados ${ }^{41}$ las niñas, niños y adolescentes en México manifiestan una alta frecuencia de aburrimiento, tristeza, incertidumbre, angustia, entre otros estados de ánimo y sensaciones. La mayoría de las y los participantes $(84 \%)$ relacionaron el encierro con la palabra aburrimiento, seguida de la sensación de tristeza con el 64\%. Nueve de cada diez niñas, niños y adolescentes sienten preocupación de que alguien de su familia se enferme o muera a causa de COVID-19. Después de la salud, su preocupación se centra en la pérdida del trabajo de las personas adultas que les rodean. Por otro lado, la palabra familia fue frecuentemente asociada con emociones positivas, como una fuente de alegría y seguridad, goce en la convivencia y sensación de protección. En el caso de niñas y niños en primera infancia - quienes participaron en la consulta por medio de dibujosllama la atención la representación del coronavirus como un personaje que ataca o se sobrepone al mundo, a la familia o a la casa. Acompañado de esto, la casa permanece como el espacio de protección.

De un análisis de cada rubro, en particular respecto al miedo y la tristeza, es posible evidenciar que la intensidad o bien la constancia de esas emociones principales y otras relacionadas, generan condiciones propicias para afectar

40 Organización de las Naciones Unidas, Comité de Derechos Económicos, Sociales y Culturales (CESCR), Observación general núm.14, El derecho al disfrute del más alto nivel posible de salud (artículo 12 del Pacto Internacional de Derechos Económicos, Sociales y Culturales), 11 de agosto de 2000, E/C.12/2000/4, disponible en: https://wrere.refworld.org. es/docid/47ebcc492.html.

41 Comisión de Derechos Humanos de la Ciudad de México, \#InfanciasEncerradas. Consulta a niñas, niños y adolescentes/Reporte nacional, Ciudad de México, 2020, disponible en: https:// cdhcm.org.mx/wp-content/uploads/2020/07/Infancias_encerradas_Nacional.pdf. 
Este libro forma parte del acervo de la Biblioteca Jurídica Virtual del Instituto de Investigaciones Jurídicas de la UNAM

la salud mental. Si bien estas emociones no son experiencias exclusivas de este grupo etario durante la pandemia, sí constituyen indicios significativos que justifican la implementación de estrategias específicas de prevención para la salud mental en el grupo de población de niñas, niños y adolescentes.

Es importante tener en cuenta que, así como existen determinantes para la salud en general, también los hay para la salud mental en específico, pues influyen múltiples factores biológicos pero también medioambientales y sociales. ${ }^{42}$ En el contexto actual, es preciso adoptar medidas de atención oportuna a estos factores e identificar su relación con la depresión o con la presencia e incremento de conductas de riesgo, entre otras. La depresión, como un ejemplo, puede aparecer desde la infancia y al comienzo de la adolescencia. ${ }^{43}$ Es importante advertirla y atenderla en su estado inicial, dado que su evolución conlleva mayores riesgos para el estado general de las personas a lo largo del ciclo de vida y afecta los diversos ámbitos de desarrollo como el académico, social, familiar e incluso puede comprometer la integridad personal. ${ }^{44}$

Como elementos importantes de contexto, no debe desestimarse el aumento en la tasa de suicidio, autolesiones y malestar entre la población. Respecto al suicidio, tan solo en la Ciudad de México, de marzo a julio de 2020 se registraron 29 suicidios correspondientes a adolescentes y niños. ${ }^{45}$ Esta cifra es alarmante si se considera que, para el mismo periodo en 2019, se registraron 14; es decir, un número significativamente menor. ${ }^{46}$

Además de la identificación de los factores, es importante acompañar el desarrollo de hábitos de higiene mental que contribuyan a fortalecer a las personas frente a las amenazas a la salud mental. Lo anterior no sólo es relevante para procurar la salud de las niñas, niños y adolescentes en su etapa de vida actual, sino que la atención temprana y el desarrollo oportuno de habilidades de autocuidado contribuyen también a un sano desarrollo de las personas en etapas de vida posteriores.

42 Organización Mundial de la Salud, Social determinants of mental health, OMS, Ginebra, Suiza, 2014, disponible en: https://apps.who.int/iris/bitstream/handle/10665/112828/ 9789241506809_eng.pdf.

43 Cohen, Joseph R. et al., "Anxiety and Depression During Childhood and Adolescence: Testing Theoretical Models of Continuity and Discontinuity", Fournal of abnormal child psychology, vol. 46, núm. 6, 2018, pp. 1295-1308, DOI:10.1007/s10802-017-0370-x.

44 Mendelson, T. y Tandon, S. D., "Prevention of Depression in Childhood and Adolescence", Child and Adolescent Psychiatric Clinics of North America, vol. 25, núm. 2, 2016, pp. 201 218, DOI:10.1016/j.chc.2015.11.005.

45 Gobierno de la Ciudad de México, Víctimas en carpetas de investigación de la Fiscalía General de Fusticia de la Ciudad de México, datos personalizados obtenidos del portal de datos abiertos, https://datos.cdmx.gob.mx/explore/dataset/victimas-en-carpetas-de-investigacion-pgj/table/.

46 Idem. 
Este libro forma parte del acervo de la Biblioteca Jurídica Virtual del Instituto de Investigaciones Jurídicas de la UNAM

Una medida que contribuye a mantener la salud emocional y física de niñas, niños y adolescentes durante este periodo de encierro es fomentar la actividad física y la promoción de estilos de vida saludables con recomendaciones específicas. ${ }^{47}$ Otro elemento importante es procurar el vínculo entre pares y mantener activa la propuesta y el acceso a actividades lúdicas y creativas. Al respecto, países como Chile que ha mantenido un estricto control sobre la población, de cualquier modo ha permitido que niños, niñas y adolescentes realicen actividades en el exterior con una persona adulta - dependiendo de la edad- algunos días de la semana. ${ }^{48}$ Asimismo, los espacios de escucha para que niñas, niños y adolescentes puedan comunicar sus experiencias ya sea a sus familiares o bien a especialistas, es fundamental.

Es importante colocar la información específica obtenida de ejercicios como la consulta \#InfanciasEncerradas y otros similares, en la ruta conducente para el diseño de medidas de atención inmediata para mejorar el bienestar mental de niñas, niños y adolescentes. Además, es importante mencionar que la lectura que puedan hacer los principales cuidadores de las infancias y adolescencias de los resultados puede servir para acompañar la vida emocional de hijos e hijas. Dicho acompañamiento por el núcleo de cuidado puede significar que se impida el progreso de tal impacto; es decir, es la principal medida de prevención.

Por lo tanto, las políticas que busquen mejorar el bienestar de niñas, niños y adolescentes deben incluir medidas dirigidas para involucrar a los adultos que les rodean. Por ejemplo, el Ministerio de Salud de Argentina ha emitido una guía dirigida a madres, padres o principales referentes de cuidado para procurar la salud mental de sus hijos e hijas en edad infantil y adolescente. ${ }^{49}$ Esta guía está basada en un documento emitido por la OMS. ${ }^{50}$

47 Guan, Hongyan et al., "Promoting healthy movement behaviours among children during the COVID-19 pandemic", The Lancet. Child \& Adolescent Health, vol. 4, núm. 6, 2020, pp. 416-418, DOI:10.1016/S2352-4642(20)30131-0.

48 Télam, "Los niños podrán salir tres veces por semana a lugares abiertos durante la cuarentena", 10 de agosto de 2020, disponible en: https://wrere.telam.com.ar/notas/202 008/ 500718-ninos-podran-para-salir-de-sus-casas-durante-la-pandemia-en-chile.html.

49 Dirección Nacional de Salud Mental y Adicciones, "Recomendaciones sobre salud mental para la población de niños, niñas y adolescentes por la pandemia COVID-19", $M i$ nisterio de Salud Argentina, Argentina, 1o. de abril de 2020, disponible en: http://wrere.msal.gob. ar/images/stories/bes/graficos/0000001886cnt-covid-19-recomendaciones-salud-mental-ninos-ninasadolescentes-contexto-pandemia.pdf.

50 Organización Mundial de la Salud, "Mental health and psychosocial considerations during the COVID-19 outbreak", 18 de marzo de 2020, disponible en: https://apps.who.int/ iris/bitstream/handle/10665/331490/WHO-2019-nCoV-MentalHealth-2020.1-eng.pdf. 
Este libro forma parte del acervo de la Biblioteca Jurídica Virtual del Instituto de Investigaciones Jurídicas de la UNAM

Pero ¿cómo lograr el cuidado del bienestar psicosocial de niñas, niños y adolescentes cuando los adultos están también sufriendo impactos graves a su propia salud mental? ¿Cómo asegurar los espacios de escucha y el desarrollo del vínculo entre pares cuando nos enfrentamos a una realidad donde muchos menores de edad tendrán que permanecer solos en casa estando las escuelas cerradas pero el mundo laboral volviendo lentamente a la normalidad?

\section{CONGLUSIONES: LA URGENGIA DE LO INMEDIATO}

Las niñas, niños y adolescentes han sido — y seguirán siendo - los protagonistas de las medidas de distanciamiento como eje de las medidas de mitigación de esta pandemia. Esto ha significado cambios drásticos a sus vidas diarias cuyo efecto no debe ser subestimado. Más allá de los impactos a la salud física de este grupo etario - aunque dichas consideraciones también son relevantes y deben ser atendidas - nos estamos enfrentando a una crisis de salud mental en este grupo etario, resultado del cambio en la rutina, las expectativas y las condiciones de encierro, el aislamiento y la creciente preocupación por la situación económica de sus padres o cuidadores, entre otros.

Debido a la incertidumbre que existe sobre tantos aspectos de nuestras vidas en sociedad, se vuelve difícil pensar en estrategias para atender los efectos a mediano y largo plazo de esta pandemia. Sin embargo, esto no es excusa para descuidar los efectos inmediatos que estamos observando, especialmente si consideramos que nuestro regreso a la normalidad sigue dependiendo de que las infancias y adolescencias no vuelvan a la suya. La implementación de medidas a corto plazo es urgente e indispensable para atender al bienestar integral de niñas, niños y adolescentes y asegurar el pleno ejercicio de sus derechos.

Cualquier estrategia debe de trascender la perspectiva exclusiva o principalmente médica para transitar hacia una perspectiva psicosocial que abarque más herramientas de diagnóstico individual y comunitario, así como que aporte medidas más amplias desde estos enfoques para procurar la integralidad del abordaje. Es decir que las estrategias de prevención y atención para la salud mental de niñas, niños y adolescentes deben incluir el fortalecimiento de las familias en su diversidad de composición, para que a su vez las niñas, niños y adolescentes encuentren seguridad y protección ante el contexto. Éstas deben cubrir los ámbitos tanto de cuidado emocional y prevención de la violencia como los relativos a la garantía de un nivel de vida adecuada. Todo lo anterior requiere apoyo dirigido tanto a las 
Este libro forma parte del acervo de la Biblioteca Jurídica Virtual del Instituto de Investigaciones Jurídicas de la UNAM

personas que fungen como principales cuidadoras, como a la población en general y a los propios niños, niñas y adolescentes para el desarrollo de estrategias de autocuidado.

Las estrategias e intervenciones deben ser interseccionales e involucrar, principalmente, el ámbito educativo y de la salud, desde un enfoque comunitario de la salud pública. Es preciso que estén diseñadas a partir de los diferentes contextos, del enfoque diferenciado e interseccional de modo que puedan cubrir la diversidad propia del grupo etario: diversidad sexogenérica, indígenas y afrodescendientes, con discapacidad, en función del género, si viven en contexto familiar o bien en una institución, entre otros.

Esta pandemia ha realzado el papel tan esencial que juegan las niñas, niños y adolescentes en nuestras sociedades. Sin su cooperación con las medidas de mitigación, imaginamos que los efectos de la crisis sanitaria serían todavía más devastadores. Por lo tanto, esperamos que así como este momento histórico nos ha hecho reflexionar sobre cómo queremos que se vea nuestro mundo en el futuro, pensemos también en cómo poner a las infancias y adolescencias al centro de las discusiones que les afectan y que sus vivencias nos dejen de parecer secundarias. 